\title{
A Note on the Pricing of Multivariate Contingent Claims Under a Transformed-Gamma Distribution
}

\author{
Luiz Vitiello* and Ivonia Rebelo
}

${ }^{*}$ Corresponding author. Luiz Vitiello is at Essex Business School, University of Essex, email: lrviti@essex.ac.uk. Ivonia Rebelo is at London Metropolitan Business School, London Metropolitan University, email: i.rebelo@londonmet.ac.uk. Please send all correspondence to Essex Business School, University of Essex, Wivenhoe Park, Colchester, CO4 3SQ, UK. We would like to thank Keith Cuthbertson, Roman Matousek and Ser-Huang Poon for valuable comments. 


\title{
A Note on the Pricing of Multivariate Contingent Claims under a Transformed-Gamma Distribution
}

\begin{abstract}
We develop a framework for pricing multivariate European-style contingent claims in a discrete-time economy based on a multivariate transformedgamma distribution. In our model, each transformed-gamma distributed underlying asset depends on two terms: a idiosyncratic term and a systematic term, where the latter is the same for all underlying assets and has a direct impact on their correlation structure. Given our distributional assumptions and the existence of a representative agent with a standard utility function, we apply equilibrium arguments and provide sufficient conditions for obtaining preference-free contingent claim pricing equations. We illustrate the applicability of our framework by providing examples of preference-free contingent claim pricing models. Multivariate pricing models are of particular interest when payoffs depend on two or more underlying assets, such as crack and crush spread options, options to exchange one asset for another, and options with a stochastic strike price in general.
\end{abstract}

Keywords: Multivariate Transformed-Gamma Distribution, Risk Neutral Valuation Relationship, Multivariate Contingent Claim, Stochastic Strike Price, General Equilibrium.

JEL classification: G13. 


\section{A Note on the Pricing of Multivariate Contingent Claims under a Transformed-Gamma Distribution}

\section{Introduction}

Derivative markets provide investors with a variety of different types of instruments for managing the risk of their portfolios. One type of derivative is related to contingent claims whose payoffs depend on more than one underlying asset, such as options to exchange one asset for another, options on mutual funds, crack and crush spread options, and options with a stochastic strike price in general. This paper focus on the pricing of such derivatives.

Most of the previous studies on the pricing of multivariate contingent claims rely, in some way or another, on the assumption of normally or transformed-normally distributed prices or cash flows i.e. they are extensions of the Black and Scholes (1973) and Brennan (1979) models. Examples

include, amongst others, Camara (2005), Johnson (1987), Margrabe (1978), Stapleton and Subrahmanyam (1984), and Stulz (1982). In this paper we depart from the well known Gaussian framework by letting the underlying assets have a multivariate transformed-gamma distribution. That is, we develop a framework for pricing multivariate European-style contingent claims in a discrete-time economy based on a multivariate transformed-gamma distribution.

In our framework, each transformed-gamma distributed underlying asset depends on two gamma-distributed terms, where one can be regarded as 
systematic and the other as idiosyncratic. While the idiosyncratic terms of the underlying assets are, of course, independent of each other, the systematic term is the same for all underlying assets and has a direct impact on their correlation structure. Also, the distributions of the underlying assets do not need to be the same, but must belong to the family of transformed-gamma distributions.

Given the distributional assumptions and the existence of a representative agent with a standard utility function, we show explicitly the link between the primitives of the economy and the underlying assets. We also show that, in certain cases, it is possible to obtain preference-free contingent claim pricing equations by using equilibrium arguments alone without having to rely on the assumption of risk neutrality. We illustrate the applicability of our framework by presenting examples of preference-free option pricing models that depend on one or more underlying assets.

Applications of the gamma distribution ${ }^{1}$ to finance include the variancegamma process of Madan and Seneta (1990) and the respective variancegamma option pricing model of Madan, Carr and Chang (1998), the gamma option pricing models of Gerber and Shiu (1994), Heston (1993) and Schroder (2004), the univariate transformed-gamma option pricing model of Vitiello and Poon (2010), the Markovian gamma model of Leobacher and Ngare (2011), and the bilateral gamma model of Bellini and Mercuri (2014) amongst others. In addition to this, the gamma distribution has been applied, for in-

\footnotetext{
${ }^{1}$ The gamma distribution contains the normal distribution as a limiting case and, as the lognormal distribution, it lies on a single line in the skewness-kurtosis plane (see Johnson et al. 1994).
} 
stance, to income distribution by Salem and Mount (1974), to insurance derivatives by Lane and Movchan (1999), and more widely to model natural events, in particular rainfall (Griffiths, 1990; Simpson, 1972), which suggests that pricing models based on the gamma distribution may be a good alternative to price such natural events.

The rest of this article is organised as follows: in Section 2 the basic economy and the multivariate transformed-gamma distribution are introduced, and the asset specific pricing kernel is obtained. In Section 3 an equilibrium relationship for the price of the underlying assets is obtained and new preference-free multivariate transformed-gamma contingent claim pricing models are derived. Section 4 concludes.

\section{The Valuation Model}

\subsection{The Economy}

The basic setting introduced in this section is similar to the economy developed by Camara (2005) and Stapleton and Subrahmanyam (1984), in which there is a representative investor who maximises her expected utility of terminal wealth, $\operatorname{Max} E[U]$, where $U$ is the investor's utility function of wealth and $E[\cdot]$ is the expectation taken with respect to the physical probability measure.

In equilibrium, it follows from the first order condition that the vector of 
current forward prices of payoffs $\mathbf{V}(\mathbf{X})$ is given by

$$
\begin{aligned}
\mathbf{P}(\mathbf{V}(\mathbf{X})) & =\frac{E\left[\mathbf{V}(\mathbf{X}) U_{W}\right]}{E\left[U_{W}\right]} \\
& =E[\mathbf{V}(\mathbf{X}) \phi(\mathbf{X})]
\end{aligned}
$$

where $U_{W}$ is the first derivative of the utility function with respect to wealth, $\mathbf{V}(\mathbf{X})$ is the vector of payoffs of the claims $\mathbf{V}(\cdot)$ as a function of the payoff of underlying assets $\mathbf{X}=\left(x_{1}, \ldots, x_{M-1}\right)$, and

$$
\phi(\mathbf{X})=\frac{E\left[U_{W} \mid \mathbf{X}\right]}{E\left[U_{W}\right]}
$$

is defined as the asset-specific pricing kernel.

It follows that the forward value of the underlying assets can also be obtained from equation (1). For instance, if $\mathbf{V}(\mathbf{X})=\mathbf{X}$ then $\mathbf{P}(\mathbf{X})=$ $E[\mathbf{X} \phi(\mathbf{X})]$, and $\mathbf{P}(\mathbf{X})$ is the vector of forward values of the underlying assets $\mathrm{X}$.

\subsection{Distributional Assumptions}

There are several ways of constructing multivariate distributions. An overview is provided by Kotz et al. (2000). Here, we apply the Mathai and Moschopoulos (1991) multivariate gamma distribution which, amongst other features, guarantees gamma distributed marginal densities. The gamma and the multivariate gamma distributions are introduced in the following definitions.

Definition 2.1 (The gamma distribution) The random variable $y$ is said to be gamma distributed, $y \sim G(p, \sigma, \mu)$, when its three-parameter density function is given by

$$
f(y ; p, \sigma, \mu)=\frac{(y-\mu)^{p-1}}{\sigma^{p} \Gamma(p)} \exp \left[-\left(\frac{y-\mu}{\sigma}\right)\right], \quad \mu<y<\infty,
$$


where $p>0$ is the shape parameter, $\sigma>0$ is the scale parameter, $\mu$ is the location parameter and $\Gamma(\cdot)$ is the gamma function.

Definition 2.2 (The multivariate gamma distribution) Let $y_{i} \sim G\left(p_{i}, \sigma_{i}, \mu_{i}\right)$, for $i=0, \ldots, M$, be mutually independent, and

$$
z_{i}=\frac{\sigma_{i}}{\sigma_{0}} y_{0}+y_{i}, \quad i=1, \ldots, M,
$$

then the vector $\mathbf{Z}=\left(z_{1}, \ldots, z_{M}\right)$ is said to have a multivariate gamma distribution. Thus, $z_{i} \sim G\left(p_{0}+p_{i}, \sigma_{i}, \mu_{i}+\mu_{0} \sigma_{i} / \sigma_{0}\right)$ as in Definition 2.1.

Given Definition 2.2, one can think of $y_{i}$ as an idiosyncratic term and $y_{0}$ as a systematic term (a disturbance on $y_{i}$ ), leading to correlated $z_{i}^{\prime} s$. Note that according to this definition the $y^{\prime} s$ are mutually independent but the $z^{\prime} s$ are positively correlated with $\operatorname{Cov}\left(z_{i}, z_{j}\right)=p_{0} \sigma_{i} \sigma_{j}, i \neq j$.

The joint density of $z_{1}, \ldots, z_{M}$ is given by

$$
\begin{aligned}
f\left(z_{1}, \ldots, z_{M}\right) & =\int_{y_{0}} \prod_{i=1}^{M} \frac{\left(y_{0}-\mu_{0}\right)^{p_{0}-1}}{\Gamma\left(p_{0}\right) \Gamma\left(p_{i}\right) \sigma_{0}^{p_{0}} \sigma_{i}^{p_{i}}}\left(z_{i}-y_{0} \sigma_{i} / \sigma_{0}-\mu_{i}\right)^{p_{i}-1} \\
& \exp \left(-\left(y_{0}-\mu_{0}\right) / \sigma_{0}-\left(z_{i}-y_{0} \sigma_{i} / \sigma_{0}-\mu_{i}\right) / \sigma_{i}\right) d y
\end{aligned}
$$

which can be obtained by using definitions 2.1 and 2.2 , and integrating with respect to $y_{0}$.

\subsection{The Asset Specific Pricing Kernel}

In this subsection we obtain a functional form of the asset-specific pricing kernel introduced in equation (2). We present below two definitions, one which introduces the distribution of terminal wealth and of the underlying 
assets' payoff, and a second definition which introduces investors' marginal utility function of wealth.

Definition 2.3 (The distribution of wealth and of the underlying assets) Terminal wealth and the payoff of the underlying assets have a multivariate transformed gamma distribution given respectively by

$$
\begin{gathered}
h_{M}(W)=h_{M}\left(z_{M}\right)=\frac{\sigma_{M}}{\sigma_{0}} y_{0}+y_{M}, \\
h_{i}\left(x_{i}\right)=h_{i}\left(z_{i}\right)=\frac{\sigma_{i}}{\sigma_{0}} y_{0}+y_{i}, \quad i=1, \ldots, M-1,
\end{gathered}
$$

where $h_{i}(\cdot)$, for $i=1, \ldots, M$ is a monotonic differentiable function, and $y_{0}$ and $y_{i}$ have a three parameter gamma distribution as in Definition 2.1.

In the definition above the functions $h_{1}\left(x_{1}\right), \ldots, h_{M}(W)$ do not need to be the same. For instance, if $h_{i}\left(x_{i}\right)=x_{i}$ then the gamma distribution obtains. If $h\left(x_{i}\right)=\ln x_{1}$ we obtain the log-gamma distribution used by Heston (1993), and if $h_{i}\left(x_{i}\right)=\sigma_{i} \exp \left[\left(x_{i}-\mu_{i}-\mu_{0} \sigma_{i} / \sigma_{0}\right) / \sigma_{i}\right]+\mu_{i}+\mu_{0} \sigma_{i} / \sigma_{0}$ and $p_{0}+p_{i}=1$ we obtain the Gumbel distribution.

Considering definitions 2.2 and 2.3 , the joint density of $x_{1}, \ldots, x_{M}$ is given by

$$
\begin{aligned}
f\left(x_{1}, \ldots, x_{M}\right) & =\int_{y_{0}} \prod_{i=1}^{M} \frac{\left(y_{0}-\mu_{0}\right)^{p_{0}-1}\left|h_{i}^{\prime}\left(x_{i}\right)\right|}{\Gamma\left(p_{0}\right) \Gamma\left(p_{i}\right) \sigma_{0}^{p_{0}} \sigma_{i}^{p_{i}}}\left(h_{i}\left(x_{i}\right)-\frac{\sigma_{i}}{\sigma_{0}} y_{0}-\mu_{i}\right)^{p_{i}-1} \\
& \exp \left(-\left(y_{0}-\mu_{0}\right) / \sigma_{0}-\left(h_{i}\left(x_{i}\right)-\frac{\sigma_{i}}{\sigma_{0}} y_{0}-\mu_{i}\right) / \sigma_{i}\right) d y
\end{aligned}
$$

Definition 2.4 (The marginal utility function) The representative investor has a marginal utility function of wealth given by $U_{W}=\exp \left[\gamma h_{M}(W)\right]$, where the constant $\gamma$ is a preference parameter. 
The transformed function $h_{M}(W)$ matches the terminal distribution of wealth with the marginal utility function of wealth. For instance, if $h_{M}(W)=$ $\ln W$, investors have a marginal power utility function and present Constant Relative Risk Aversion (CRRA); if $h_{M}(W)=W$, then investors present a marginal exponential utility function with Constant Absolute Risk Aversion (CARA).

The previous three definitions lead to Proposition 2.5 below, where the functional form of the asset-specific pricing kernel is formally introduced.

Proposition 2.5 (The asset specific pricing kernel) Given definitions 2.2, 2.3 and 2.4, the asset-specific pricing kernel is given by

$$
\begin{aligned}
\phi(\mathbf{X}) & =\frac{\left(1-\gamma \sigma_{M}\right)^{p_{0}}}{f\left(x_{1}, \ldots, x_{M-1}\right)} \int_{y_{0}} e^{y_{0}^{*}\left(\gamma \sigma_{M}-1\right)} \frac{\left(y_{0}^{*}\right)^{p_{0}-1}}{\Gamma\left(p_{0}\right)} \\
& \prod_{i=1}^{M-1} \frac{\left|h_{i}^{\prime}\left(x_{i}\right)\right|}{\Gamma\left(p_{i}\right) \sigma_{i}^{p_{i}}}\left(h_{i}\left(x_{i}\right)-\sigma_{i} y_{0}^{*}-\mu_{i}^{*}\right)^{p_{i}-1} \\
& \exp \left[-\left(h\left(x_{i}\right)-\sigma_{i} y_{0}^{*}-\mu_{i}^{*}\right) / \sigma_{i}\right] d y_{0},
\end{aligned}
$$

where $\mathbf{X}=\left(x_{1}, \ldots, x_{M-1}\right), y_{0}^{*}=\left(y_{0}-\mu_{0}\right) / \sigma_{0}, \mu_{i}^{*}=\mu_{i}+\mu_{0} \sigma_{i} / \sigma_{0}$, and $f\left(x_{1}, \ldots, x_{M-1}\right)$ is given by equation (8).

Proof. See Appendix.

This result is used in the next section to price the underlying assets $x_{1}, x_{2}, \ldots, x_{M-1}$, and the respective contingent claims written on them.

\section{Prices in Equilibrium}

The pricing equation in (1) can be used to price any specific asset in $\mathbf{X}$. Thus, considering equation (5), the equilibrium relationship for the forward 
price of the underlying assets, where $V\left(x_{i}\right)=x_{i}$, can be written as

$$
\begin{aligned}
P\left(x_{i}\right) & =E\left[x_{i} \phi(\mathbf{X})\right] \\
& =\int_{x_{1}} \cdots \int_{x_{M-1}} x_{i} \phi(\mathbf{X}) f\left(x_{1}, \ldots, x_{M-1}\right) d x_{1} \ldots d x_{M-1},
\end{aligned}
$$

where the density $f\left(x_{1}, x_{2}, \ldots, x_{M-1}\right)$ is given by equation (8).

Similarly, the current forward price of any contingent claim with a payoff $V(\mathbf{X})$ is given by

$$
\begin{aligned}
P(V(\mathbf{X})) & =E[V(\mathbf{X}) \phi(\mathbf{X})] \\
& =\int_{x_{1}} \ldots \int_{x_{M-1}} V\left(x_{1}, \ldots, x_{M-1}\right) \\
& \phi(\mathbf{X}) f\left(x_{1}, \ldots, x_{M-1}\right) d x_{1} \ldots d x_{M-1} .
\end{aligned}
$$

If equation (10) has a solution then it may be possible to solve for the preference parameters and substitute the resulting expression into equation (11), which makes possible to eliminate preference parameters from the option pricing equation. Thus, by replacing the preference parameter by a certain function of the current forward price of the underlying assets, $P(\mathbf{X})$, it is possible to achieve risk neutrality, such that $P(V(\mathbf{X}))=E[V(\mathbf{X}) \phi(\mathbf{X})]=$ $E^{Q}[V(\mathbf{X})]$, where $E^{Q}$ means that the expectation is taken with respect to the risk-neutral density. This result can be used to obtain preference free European-style contingent claim equations. A few examples are provided below. ${ }^{2}$

Example 3.1 (The gamma vanilla call option) We are interested in a call option written on the payoff of a single log-gamma distributed underlying

\footnotetext{
${ }^{2}$ The equations presented in the examples can be solved numerically. For a survey of numerical methods for solving this type of equation see Carmona and Durrleman (2003).
} 
asset $V(x)=x_{1}$, where $h_{1}\left(x_{1}\right)=x_{1}$. The equilibrium forward price of $x_{1}$ can be obtained by using equation (10) for $i=1$, Definition 2.3, and Proposition 2.5 as follows,

$$
\begin{aligned}
P\left(x_{1}\right) & =E\left[x_{1} \phi\left(x_{1}\right)\right]=\int_{x_{1}} x_{1} \phi\left(x_{1}\right) f\left(x_{1}\right) d x \\
& =\mu_{1}^{*}+\sigma_{1} p_{1}+\frac{\sigma_{1} p_{0}}{1-\gamma \sigma_{M}},
\end{aligned}
$$

where $f\left(x_{1}\right)$ comes from equation (5).

In this case it is possible to use the forward equilibrium relationship above to solve for the preference parameter $\gamma$, which yields

$$
\gamma=\left(1-\frac{\sigma_{1} p_{0}}{P\left(x_{1}\right)-\sigma_{1} p_{1}-\mu_{1}^{*}}\right) \frac{1}{\sigma_{M}} .
$$

As discussed above, equation (13) shows that the preference parameter can be expressed as a function of the price of the underlying assets. This allows us to obtain the forward price of contingent claims in a preference free form such that $P\left(V\left(x_{1}\right)\right)=E^{Q}\left[V\left(x_{1}\right)\right]$. For instance, for a call option with payoff $V\left(x_{1}\right)=\operatorname{Max}\left(x_{1}-K, 0\right)$ and strike price $K$ its forward price can be obtained by the following specific form of equation (11)

$$
\begin{aligned}
P\left(V\left(x_{1}\right)\right) & =E\left[V\left(x_{1}\right) \phi\left(x_{1}\right)\right] \\
& =\int_{x_{1}} \operatorname{Max}\left(x_{1}-K, 0\right) \phi\left(x_{1}\right) f\left(x_{1}\right) d x_{1},
\end{aligned}
$$

where $f\left(x_{1}\right)$ is given by equation (5). Finally, by replacing the preference parameter $\gamma$ in the equation above by the relationship given in equation (13), one obtains a preference free option pricing formula.

Example 3.2 (The option to exchange an underlying asset for another: the gamma- $\chi^{2}$ call option) The value of an option to exchange one underlying 
asset for another based on the Black and Scholes (1973) equation was first proposed by Margrabe (1978). Here we present a solution for the price of a call option to exchange a gamma distributed variable $x_{1}$ for a $\chi^{2}$ distributed variable $x_{2}$ with payoff function given by $V\left(x_{1}, x_{2}\right)=\operatorname{Max}\left(x_{1}-x_{2}-K, 0\right)$. We let $h_{1}\left(x_{1}\right)=x_{1}$ and $h_{2}\left(x_{2}\right)=\left[\left(x_{2}-\mu_{2}^{*}\right) / 2+\mu_{2}^{*}\right]$ with $p_{0}+p_{2}=p$.

The results obtained in the previous example can be used for $x_{1}$. The equilibrium price of $x_{2}$ can be obtained through the application of equation (10), which yields

$$
P\left(x_{2}\right)=2 \sigma_{2}\left(\frac{p}{2}-p_{0}\right)+\frac{2 \sigma_{2} p_{0}}{\left(1-\gamma \sigma_{M}\right)}+\mu_{2}^{*}
$$

As before, solving for the preference parameter leads to the following relationship

$$
\gamma=\frac{1}{\sigma_{M}}\left[1-\frac{2 \sigma_{2} p_{0}}{P\left(x_{2}\right)-2 \sigma_{2}\left(\frac{p}{2}-p_{0}\right)-\mu_{2}^{*}}\right],
$$

which, in equilibrium, must be equal to equation (13).

The current forward price of a contingent claim with payoff $V\left(x_{1}, x_{2}\right)$ can be obtained by using equation (11), yielding

$$
\begin{aligned}
P\left(V\left(x_{1}, x_{2}\right)\right) & =E\left[V\left(x_{1}, x_{2}\right) \phi\left(x_{1}, x_{2}\right)\right] \\
& =\int_{x_{2}} \int_{x_{1}} \operatorname{Max}\left(x_{1}-x_{2}-K, 0\right) \phi\left(x_{1}, x_{2}\right) f\left(x_{1}, x_{2}\right) d x_{1} d x_{2} .
\end{aligned}
$$

In order to solve the above equation, we use conditional expectations such 
that

$$
\begin{aligned}
P\left(V\left(x_{1}, x_{2}\right)\right) & =E\left[V\left(x_{1}, x_{2}\right) \phi\left(x_{1}, x_{2}\right)\right] \\
& =E\left[E\left[V\left(x_{1}, x_{2}\right) \phi\left(x_{1}, x_{2}\right) \mid x_{2}\right]\right] \\
& =\int_{x_{2}} E\left[V\left(x_{1}, x_{2}\right) \phi\left(x_{1}, x_{2}\right) \mid x_{2}\right] f_{2}\left(x_{2}\right) d x_{2},
\end{aligned}
$$

where $f_{2}\left(x_{2}\right)$ and $\phi\left(x_{1}, x_{2}\right)$ are given by equations (8) and (9) respectively, and the conditional expectation is $f\left(x_{1} \mid x_{2}\right)=f\left(x_{1}, x_{2}\right) / f\left(x_{2}\right)$. Replacing the preference parameters in equation (18) by equations (13) and (16), results in a preference-free option pricing formula.

The examples above prove the following proposition.

Proposition 3.3 (Sufficient condition for preference free option pricing formulae) If the payoff of the underlying assets have a distribution according to Definition 2.3, the preference parameter in equation (10) can be written as a function of the underlying variables and distributional parameters, and the asset specific pricing kernel in Proposition 2.5 holds then a risk neutral valuation relationship exists.

\section{Conclusion}

In this paper we develop a framework for the pricing of multivariate contingent claims based on a transformed multivariate gamma distribution, extending the work of Camara (2005) and Stapleton and Subrahmanyam (1984) to a non-Gaussian setting. The resulting contingent claim pricing equations are 
free of preference parameters. ${ }^{3}$

The gamma distribution has been widely applied to model natural events, rainfall in particular. The framework developed here could help the pricing of contingent claims on such situations, for instance. ${ }^{4}$ Also, it could also be useful for pricing multivariate commodity options, as it can capture the increasing implied volatility pattern of such assets (see Vitiello and Poon, 2010; Zhou, 1998). Thus one could price an option written several agricultural commodities, such as crush spread options.

\footnotetext{
${ }^{3}$ The applicability of equations obtained here depends on the estimation of the relevant parameters. Depending on the information available, the method of moments can applied or the methodology suggested by Mathai and Moschopoulos (1991) for instance. Alternatively one can use the parameters implied by option market prices (see for instance Mayhew, 1995; Poon and Granger, 2003).

${ }^{4}$ It is important to note that in an incomplete market setting, such as the one related to flooding, the asset specific pricing kernel in equation (9) may not be unique.
} 


\section{Appendix}

Proof. (Proposition 2.5) In order to obtain a functional form for the asset specific pricing kernel in equation (2) definitions 2.2, 2.3 and 2.4, and equation (5) are required.

The numerator in equation (2), $E\left[U_{W} \mid \mathbf{X}\right]$, can be obtained directly from the definition of the conditional distribution, $f(W \mid \mathbf{X})=f\left(x_{1}, \ldots, x_{M}\right) \div$ $\left(x_{1}, \ldots, x_{M-1}\right)$, which yields

$$
\begin{aligned}
E\left[U_{W} \mid \mathbf{X}\right] & =E\left[\exp \left(\gamma h_{M}(W)\right) \mid x_{1}, \ldots, x_{M-1}\right] \\
& =\frac{1}{f\left(x_{1}, \ldots, x_{M-1}\right)} \int_{y_{0}} \frac{e^{\gamma \mu_{M}^{*}}\left(y_{0}^{*}\right)^{p_{0}-1} e^{y_{0}^{*}\left(\gamma \sigma_{M}-1\right)}}{\Gamma\left(p_{0}\right)\left(1-\gamma \sigma_{M}\right)^{p_{M}}} \\
& \prod_{i=1}^{M-1} \frac{\left|h_{i}^{\prime}\left(x_{i}\right)\right|}{\Gamma\left(p_{i}\right) \sigma_{i}^{p_{i}}}\left(h_{i}\left(x_{i}\right)-\sigma_{i} y_{0}^{*}-\mu_{i}^{*}\right)^{p_{i}-1} \\
& \exp \left[-\left(h_{i}\left(x_{i}\right)-\sigma_{i} y_{0}^{*}-\mu_{i}^{*}\right) / \sigma_{i}\right] d y_{0},
\end{aligned}
$$

where $f\left(x_{1}, \ldots, x_{M-1}\right)$ is given by equation (8), $y_{0}^{*}=\left(y_{0}-\mu_{0}\right) / \sigma_{0}$, and $\mu_{i}^{*}=\mu_{i}+\mu_{0} \sigma_{i} / \sigma_{0}$ for $i=1, \ldots, M$.

The denominator in equation (2) can be obtained by solving the expectation $E\left[U_{W}\right]$, with $x_{M} \sim G\left(p_{0}+p_{M}, \sigma_{M}, \mu_{M}+\mu_{0} \sigma_{M} / \sigma_{0}\right)$, which yields

$$
E\left[U_{W}\right]=E\left[\exp \left(\gamma h_{M}(W)\right)\right]=\frac{\exp \left(\gamma \mu_{M}^{*}\right)}{\left(1-\gamma \sigma_{M}\right)^{p_{M}+p_{0}}} .
$$

Finally, substituting equations (A.2) and (A.1) into equation (2) yields equation (9). 


\section{References}

[1] Bellini, F., \& Mercuri, L. (2014). Option pricing in a conditional bilateral gamma model. Central European Journal of Operations Research, 22, 373-390.

[2] Black, F., \& Scholes, M. (1973). The pricing of options and corporate liabilities. Journal of Political Economy, 81, 637-654.

[3] Brennan, M. (1979). The pricing of contingent claims in discrete time models. Journal of Finance, 34, 53-68.

[4] Camara, A. (2005). Option prices sustained by risk-preferences. Journal of Business, 78, 1683-1708.

[5] Carmona, R. \& Durrleman, V. (2003) Pricing and hedging spread options, Siam Review, 45, 627-685

[6] Gerber, H., \& Shiu, E. (1994). Option pricing by Esscher transforms. Transactions of Society of Actuaries, 46, 99-140.

[7] Griffiths, G. (1990). Rainfall deficits: distribution of monthly runs. Journal of Hydrology, 115, 219-229.

[8] Heston, S. (1993). Invisible parameters in option prices. Journal of Finance, 48, 933-947.

[9] Johnson, H. (1987). Options on the maximum of the minimum of several assets. Journal of Financial and Quantitative Analysis, 22, 277-283. 
[10] Johnson, N. L., Kotz, S., \& Balakrishnan, N. (1994). Continuous univariate distributions (Vol. 1), Wiley.

[11] Kotz, S., Balakrishnan, N., \& Johnson, N. L. (2000). Continuous multivariate distributions (Vol. 1), Wiley.

[12] Lane, M., \& Movchan, O. (1999). The perfume of a premium II. Derivatives Quarterly, 5, 27-40.

[13] Leobacher, G. \& Ngare, P. (2011) On modelling and pricing rainfall derivatives with seasonality, Applied Mathematical Finance, 18, 71-91.

[14] Madan, D. B., \& Seneta, E. (1990). The variance gamma (V.G.) model for share market returns. Journal of Business, 63, 511-524.

[15] Madan, D. B., Carr, P., \& Chang, E. (1998). The variance gamma process and option pricing. European Finance Review, 2, 79-105.

[16] Margrabe, W. (1978). The value of an option to exchange one asset for another. Journal of Finance, 33, 177-186.

[17] Mathai, A. M., \& Moschopoulos, P. G. (1991). On a multivariate gamma. Journal of Multivariate Analysis, 39, 135-153.

[18] Mayhew, S. (1995). Implied volatility. Financial Analysts Journal, JulAug, 8-20.

[19] Poon, S., \& Granger, C. (2003). Forecasting volatility in financial markets: a review. Journal of Economic Literature, 41, 478-539. 
[20] Salem, A. B., \& Mount., T. D. (2005). A convenient descriptive model for income distribution: the gamma density. Econometrica, 42, 11151128.

[21] Schroder, M. (2004). Risk-neutral parameters shifts and derivatives pricing in discrete time. Journal of Finance, 59, 2375-2401.

[22] Simpson, J. (1972). Use of gamma distribution in single-cloud rainfall analysis. Monthly Weather Review, 100, 309-312.

[23] Stapleton, R. C., \& Subrahmanyam, M. G. (1984). The valuation of multivariate contingent claims in discrete time models. Journal of Finance, 39, 207-228.

[24] Stulz, R. (1982). Options on the minimum or the maximum of two risky assets: analysis and applications. Journal of Financial Economics, 10, $161-185$.

[25] Vitiello, L., \& Poon, S. (2010). General equilibrium and preference free model for pricing options under transformed Gamma. Journal of Futures Markets, 30, 409-431.

[26] Zhou, Z. (1998). An equilibrium analysis of hedging with liquidity constraints, speculation, and government price subsidy in a commodity market. Journal of Finance, 53, 1705-1736. 